\title{
EL MODELO DE ÍNDICE GDA COMO UNA HERRAMIENTA PARA MEDIR EL GRADO DE DEPENDENCIA ALIMENTARIA ${ }^{1}$
}

\author{
LOGISTIC POTENTIAL OF LATIN AMERICA AND THE CARIBBEAN COUNTRIES: AN ANALYSIS \\ BASED ON ECONOMIC, SOCIAL AND ENVIRONMENTAL INDICATORS \\ O MODELO DE ÍNDICE GDA COMO UMA FERRAMENTA PARA MEDIR O GRAU DE \\ DEPENDÊNCIA ALIMENTAR \\ Jhon Jairo Monje Carvajal ${ }^{2}$, Marlin Carolina Ortega Muñoz ${ }^{3}$, Daniel Andrés Vega Castro ${ }^{4}$
}

Fecha de recibido: Agosto 05 de 2017 | Fecha de aprobado: Octubre 05 de 2017

\section{Resumen}

La alimentación es uno de los elementos más sensibles en la evolución de la humanidad. Esta está ligada al desarrollo cultural y la adaptación del humano en el territorio, el aprovechamiento de recursos naturales para su sustento y la capacidad de ver, entender, interpretar y ajustar sus modelos de vida a las diferentes ofertas que el mismo territorio entrega. Medir la capacidad de alimentación y sustento propio es el paradigma de los últimos tiempos, pero no es fácil encontrar un índice que lo permita, por lo cual desde diferentes proyectos se ha venido evaluando un índice que permite ver qué tan local o global es la alimentación de una persona, familia o comunidad. El modelo de índice GDA tiene como objetivo brindar una herramienta que permita gestar indicadores cuantitativos de qué tan local o global es nuestra alimentación, utilizándose con éxito en diferentes comunidades del país, con resultados que han permitido generar apuestas integradoras para mitigar la dependencia alimentaria.

Palabras clave: Alimentación, sustento propio, dependencia alimentaria, consumo local, consumo global, soberanía alimentaria.

1 Artículo de reflexión que muestra de forma crítica el uso de un indicador numérico para las dependencias de la alimentación de las personas.

2 Ingeniero agroecólogo de la Universidad de la Amazonia, magíster en agroecología, sociología y desarrollo rural sustentable de la Universidad Internacional de Andalucía, doctor en recursos naturales y gestión sostenible de la Universidad de Córdoba en España. Profesor Asociado grado 2 de la Corporación Universitaria Minuto de Dios, líder del semillero de Desarrollo Rural del Grupo de Investigación Agroeco y Gestión Ambiental. Correo electrónico: jmonje@uniminuto.edu.co, jhonmonje@gmail.com

3 Ingeniera agroecóloga de la Universidad de la Amazonia, magíster en agroecología, un enfoque para la sustentabilidad rural de la Universidad de Córdoba en España. Coordinadora académica del programa de Ingeniería Agroecológica de Corporación Universitaria Minuto de Dios. Integrante del semillero de Desarrollo Rural del Grupo de Investigación Agroeco y Gestión Ambiental. Correo electrónico: marlin.ortega@uniminuto.edu, carola98818@hotmail.com.

4 Ingeniero agroecológico de la Corporación Universitaria Minuto de Dios, magíster en educación de la UNIMINUTO, maestro en educación del Politécnico de Monterrey, México. Profesor asistente 2, coordinador del programa de Tecnología en Producción Agroecológica de UNIMINUTO VIRTUAL Y DISTANCIA. investigador junior, miembro del grupo de investigación Enlaces Pedagógicos. Correo electrónico: davega@uniminuto.edu, agroecologiavega@gmail.com. 


\section{Abstract}

Food is one of the most sensitive elements in the evolution on humankind. It is linked to the cultural development and adaptation of human beings to the territory, the use of natural resources for their sustenance, and the ability to show, understand, interpret, and adjust their life forms to whatever possibilities the same territory offers. Measuring food capacity and proper sustenance is the contemporary paradigm. However, it is not easy to find an index that allows for it. Therefore, recent projects have focused on evaluating an index that can be used to determine how local or global is a person's, family's, or community's food. The purpose of the GDA-model index is to provide a tool to create quantitative indicators of the local or global nature of what we eat. It has been successfully used in different communities in Colombia. The results have allowed posing comprehensive proposals to mitigate food dependency.

Keywords: Food, proper sustenance, food dependence, local consumption, global consumption, food sovereignty.

\section{Resumo}

A alimentação é um dos elementos mais sensíveis na evolução da humanidade. Ela está unida ao desenvolvimento cultural e a adaptação do humano no território, o aproveitamento de recursos naturais para seu sustento e a capacidade de ver, entender, interpretar e ajustar seus modelos de vida às diferentes ofertas que o mesmo território oferece. Medir a capacidade de alimentação e sustento próprio é o paradigma dos últimos tempos, mas não é fácil encontrar um índice que o permita, pelo qual desde diferentes projectos se veio avaliando um índice que permite ver que tão local ou global é a alimentação de uma pessoa, família ou comunidade. O modelo de índice GDA tem como objectivo brindar uma ferramenta que permita gestar indicadores quantitativos de qué tão local ou global é nossa alimentação, se utilizando com sucesso em diferentes comunidades do país, com resultados que têm permitido gerar apostas integradoras para mitigar a dependência alimentar.

Palavras-chave: alimentação, sustento próprio, dependência alimentar, consumo local, consumo global, soberania alimentar. 


\section{INTRODUCCIÓN}

La evaluación de la sustentabilidad como un elemento integrador para entender las formas y modelos de producción desde la agroecología ha tenido un gran desarrollo en los últimos años, especialmente para construir metodologías de indicadores que permitan visibilizar los niveles de trabajo agroecológico a nivel de sistemas productivos.

La sustentabilidad, entonces, se convierte en el tema de mayor relevancia en nuestro tiempo, especialmente cuando de esta se deriva la interpretación de la capacidad de aportar a la agroecología (Leff, 2002). Además, transforma el mundo en una visión de complejidad (Morin, 1999), porque radica nuevamente la ley ecológica de las interrelaciones de aportes, consumos e impactos, que nunca estarán aisladas (Odum \& Barrett, 2008). Pero, a su vez, también es importante entender que lo que a todos nos tiene en este proceso de valoración, evaluación, crítica, y discurso, tanto técnico como político, son los elementos y formatos que involucra la alimentación de las personas en todo el planeta (Figueroa Pedraza, 2003). No es diferente el tema desde lo productivo, porque a pesar de que se habla de daños ambientales, efectos de contaminación, efectos nocivos para la salud humana y animal, etc., resultado de la aplicación del modelo de revolución verde, también implica entender que el valor político del modelo agroecológico es la alimentación.

Entonces, la alimentación toma el papel preponderante en los fundamentos y criterios de agroecología, porque referencia discursos en torno a la seguridad alimentaria y los riesgos de que el proceso de alimentación bajo este concepto en sí tome una tendencia de poder (Alonso Mielgo \& Sevilla Guzmán, 1995), de dominio de los alimentos, y esto implique el sometimiento de comunidades enteras. Es así como se indica desde la agroecología que se debe potenciar el uso de lo local, en donde el desarrollo endógeno juega un papel importante, y depender en la menor medida de los flujos externos (Godard, Ceron, Vinaver \& Passaris, 1987). Es decir, potenciar los flujos internos y minimizar las entradas externas como un elemento importante para el desarrollo local.

Desde este enfoque, la alimentación de base local se convierte en el elemento articulador del desarrollo endógeno porque potencia la dinámica interna y resalta diferentes dimensiones de los actores que la practican, lo que genera apropiación de prácticas sociales, rescate de labores culturales, conocimientos ancestrales, saberes, semillas, procesos, etc. (Martí Sanz, 2006). Pero también es importante entender y visibilizar los modelos tradicionales de producción, apropiación y consecución de alimentos, pues estos todavía constituyen la base de la alimentación de la población rural en América Latina, los cuales, después de la segunda Guerra Mundial, fueron objeto de una campaña para modificarlos, buscando su integración al modelo agroindustrial global, fundamentalmente. Estos procesos fueron promovidos por agencias internacionales, o los mismos Estados y, por supuesto, diferentes multinacionales agroalimentarias, que ven en ellos su mayor negocio y renta (Martí Sanz, 2006).

La campaña incentiva modificaciones encaminadas fundamentalmente al cambio de visión, a la reforma de las pautas de producción, transformación y comercialización de los alimentos, que hasta ese momento se hacían mayormente con recursos locales, al uso de insumos externos, especialmente de la industria de agroquímicos, de semillas, y de herramientas; de integración a modelos de mercados de superficies o grandes superficies. Esto, con el objetivo de inducir una especialización de producción con pérdida de diversidad local, logrando colocar al mismo tiempo en los mercados locales alimentos y productos del mercado mundial a precios muy bajos. Pero, a su vez, esto aumentaba el gasto de las familias en su alimentación, producía la baja en los consumos de base local y cambiaba el flujo de recursos económicos que circulaban de base local a modelos globalizados de economía (Dabat, 2002).

Hoy en día, entender las dinámicas locales de alimentación es la preocupación de instituciones que trabajan desde la agroecología, pero no es fácil determinar qué tan local, regional, nacional o global es la alimentación de una familia, comunidad o región, pues se convierte en una estrategia que involucra muchos factores, que no son tan fáciles de considerar. Por eso, hemos probado un modelo de índice de la dependencia alimentaria GDA, a partir de una metodología basada en la comida diaria, y generando un análisis de los lugares de donde provienen dichos alimentos. Por tal motivo, el modelo de índice GDA es una herramienta metodológica de construcción propia de los autores, que permite evaluar el grado de dependencia alimentaria de una persona, una familia, una comunidad o una región y cuyo funcionamiento se sustenta en una serie de preguntas sencillas asociadas a la ingesta de alimentos durante un periodo específico. Dicha herramienta relaciona un modelo de consecución de datos y origen que, al aplicar una tabla de correlaciones numéricas equivalentes, y mediante una fórmula 
matemática, permite establecer un valor cuantitativo en una escala de 1 a 5 . Cuando en el resultado obtenido los valores se aproximan a 1 el consumo es de base local, y cuanto los valores derivados del proceso más se aproximan a 5 , es de base global. la importancia del consumo de base local, rescatar los aportes que se hacen desde este a la dinámica económica de las comunidades o de una población o región, así como la disminución de la huella ecológica global por la contaminación que se hace al transportar a grandes distancias de alimentos, con un consumo increíble de combustibles derivados del petróleo. También, los aportes a la conservación de la biodiversidad local, el respeto por la cultura y la autonomía de los pueblos en cuanto a su alimentación y sustento propio.

\section{LA METODOLOGÍA DEL GDA}

La metodología sobre la cual funciona el modelo GDA funciona en tres (3) etapas. La primera consiste en ubicar el posible punto de origen de la comida que se ha consumido (se trabaja con los alimentos preparados y consumidos, no con los que se piensan o se van a consumir). La segunda etapa es la valoración del modelo del mercado en el que se apropian o consiguen los productos. La tercera es la aplicación de la fórmula matemática para obtener el índice.

En la primera etapa el modelo se maneja un globo (figura 1) que tiene dos tipos de dimensiones: una circular, que corresponden a seis niveles ${ }^{5}$, y una segunda dimensión que corresponde a los cuadrantes ${ }^{6}$.
La expectativa de este modelo es ayudar a entender

Figura 1. Globo de nivel de origen y cuadrante de recorrido

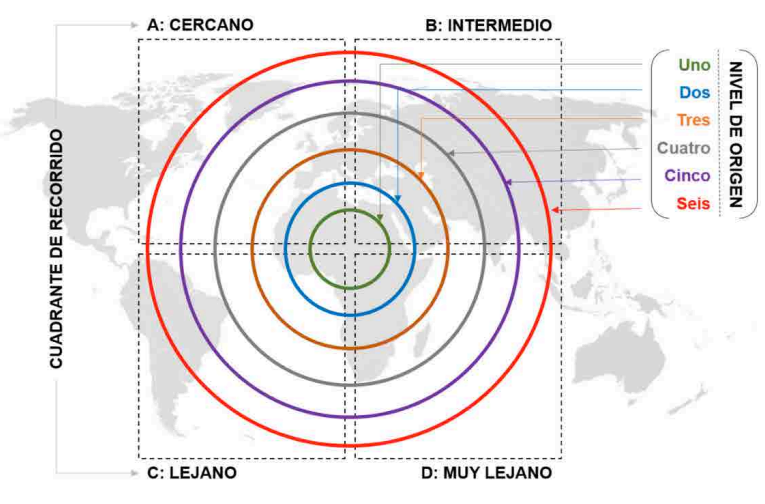

Fuente: elaboración propia.

La anterior figura indica, entonces, que un producto tiene dos coordenadas para ser ubicadas en el gráfico. La primera corresponde a la validación del alimento consumido desde local, zonal, regional, nacional, continental o mundial (tabla 1). Pero existe también la posibilidad de que el producto llegue de otra parte del mundo o de región y, por consiguiente, su recorrido es el más largo que pueda realizar dentro de ese nivel de origen, lo que da como resultado una localización en el cuadrante $\mathrm{D}$, por ser de una zona muy lejana.

Tabla 1. Relación de niveles según su origen

\begin{tabular}{|l|l|l|}
\hline NIVEL & TIPOLOGÍA & \multicolumn{1}{c|}{ RELACIÓN } \\
\hline 1 & Local & $\begin{array}{l}\text { Productos del mismo municipio, } \\
\text { localidad, población, bien sea } \\
\text { de la parte urbana, periurbana o } \\
\text { rural. }\end{array}$ \\
\hline 2 & Zonal & $\begin{array}{l}\text { De la misma zona, municipios } \\
\text { cercanos, especialmente vecinos } \\
\text { de la parte urbana, periurbana o } \\
\text { rural de dichos municipios o po- } \\
\text { blaciones. }\end{array}$ \\
\hline 3 & Regional & $\begin{array}{l}\text { Del mismo departamento, estado } \\
\text { o región, que puede ser de la } \\
\text { parte urbana, periurbana o rural. }\end{array}$ \\
\hline 4 & Nacional & $\begin{array}{l}\text { Del mismo país. } \\
\text { Del mismo continente. }\end{array}$ \\
\hline 5 & Continental & $\begin{array}{l}\text { Del male } \\
\text { De cualquier parte del mundo, } \\
\text { pero de diferente continente al } \\
\text { continente destino. }\end{array}$ \\
\hline 6 & Mundial &
\end{tabular}

Fuente: elaboración propia. sumidos, y que van desde lo local a lo global.

6 Cuatro (4) cuadrantes, uno por la distancia de recorrido de cada alimento consumido y que van de los lugares más cercanos a los más lejanos: A, alimento conseguido (producido) en un lugar cercano; $\mathrm{B}$, alimento conseguido (producido) en un lugar intermedio; $C$, alimento conseguido (producido) en un lugar lejano, y $\mathrm{D}$, alimento conseguido (producido) en un lugar muy lejano. 
(Kalmanovitz, 2003). Este plátano lo produce una persona que tiene una finca al final del pueblo, le toma menos de media hora llevarlo hasta la casa de quien lo consume. ¿Cómo tomar parámetros en el globo para dibujar sus coordenadas? Como la finca queda en el pueblo, muy cerca del lugar de destino de quien lo va a consumir, se ubica en el nivel número 1 , y como el recorrido es muy corto respecto sw los límites de la población, se ubica en el cuadrante A.

Se debe tener presente que un producto en cualquier nivel puede llegar a tener cuatro opciones de ubicación según el cuadrante. En primera medida, puede ser un producto local, pero con origen D -muy lejano-, porque se produce en los límites o partes más alejadas del nivel 1 ; o C -lejano-, porque se produce en un lugar lejano del municipio; o B -intermedio-, porque se produce en un lugar no tan apartado del mismo municipio; o A -cercano-, porque se produce en un lugar contiguo del mismo municipio. La distancia se toma de acuerdo al lugar en donde se consumió el producto y es igual para cada nivel de origen.

Figura 2. Coordenadas del producto ejemplo

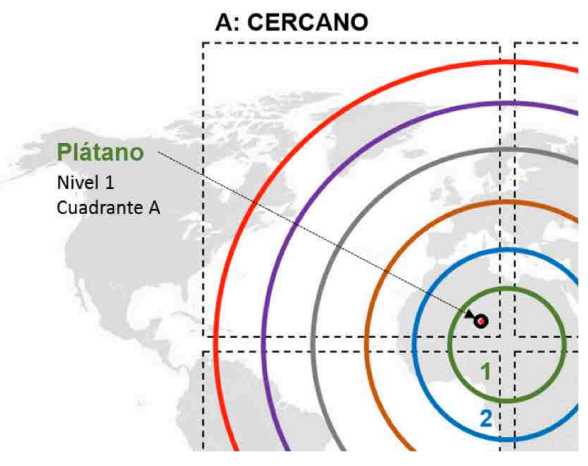

Fuente: elaboración propia.

La segunda etapa del modelo tiene dos pasos. El primero asigna puntos según la ubicación en el nivel y el cuadrante del globo (tabla 1), que para el ejemplo del producto en mención -plátano-, tendría una valoración de 10 puntos -6 puntos por estar en el nivel $1, y$ cuatro puntos por estar en el cuadrante A-. Este conteo de puntos se debe ir guardando para al final del proceso poder realizar los cálculos para la obtención del índice GDA. En este caso, la cuenta que llevamos del ejemplo son 10 puntos, que corresponden a un solo producto alimenticio.

En el segundo paso de la etapa dos, se deben considerar el tipo de mercado o comercio usado para obtener el producto alimentario, y este va de acuerdo a si se produce, se cambia en un trueque (Tocancipá, 2008), se consigue por medio de una moneda social o un tipo de mercado que no use dinero convencional (Gisbert Quero, 2013), o si simplemente se compra con dinero en cualquiera de sus formas, bien sea efectivo, dinero electrónico o tarjetas de crédito. Según sea el modelo de mercado, se asignan puntos (tabla 2), los cuales se deben restar a la cuenta que se trae del paso número uno de la etapa dos.

Tabla 2. Valoración de puntos según las coordenadas en el globo

\begin{tabular}{|c|c|c|c|}
\hline \multicolumn{2}{|c|}{ PUNTOS SEGÚN NIVEL } & \multicolumn{2}{c|}{ PUNTOS SEGÚN CUADRANTE } \\
\hline NIVEL & PUNTOS & CUADRANTE & PUNTOS \\
\hline 1 & 6 & A & 4 \\
\hline 2 & 5 & B & 3 \\
\hline 3 & 4 & C & 2 \\
\hline 4 & 3 & D & 1 \\
\hline 5 & 2 & & \\
\hline 6 & 1 & & \\
\hline
\end{tabular}

Fuente: elaboración propia.

En el ejemplo, el producto -plátano- trae una suma de puntos que equivale a 10 por el resultado de su colocación en el globo, y dicho producto fue intercambiado por quien lo consumió, lo cual lo ubica como un producto de intercambio, lo que refiere que a los 10 puntos que traía se le debe restar 1 punto según la tabla 3 . Esto quiere decir que dicho producto tiene una valoración de 9 puntos.

Tabla 3. Valoración de puntos a restar según su modelo de mercado

\begin{tabular}{|c|c|c|}
\hline TIPO & PUNTOS & \multicolumn{1}{c|}{ DESCRIPCIÓN } \\
\hline Produce & 0 (cero) & $\begin{array}{l}\text { El producto que ha consumido } \\
\text { es producido por él mismo, no } \\
\text { ha requerido ningún proceso de } \\
\text { comercio. }\end{array}$ \\
\hline Intercambia & 1 (uno) & $\begin{array}{l}\text { Lo intercambia en un mercado } \\
\text { de trueque, o ha usado una mo- } \\
\text { neda social, o un tipo de pago } \\
\text { diferente al modelo convencio- } \\
\text { nal crematístico. }\end{array}$ \\
\hline Compra & 2 (dos) & $\begin{array}{l}\text { Lo ha comprado con dinero } \\
\text { convencional, en cualquiera de } \\
\text { sus formas de pago (efectivo, } \\
\text { dinero electrónico o tarjetas de } \\
\text { crédito. }\end{array}$ \\
\hline
\end{tabular}

Fuente: elaboración propia. 
La forma de aplicar el índice en trabajos grupales con la comunidad puede tener sus variables, pues la metodología puede ir desde listados de la comida, de uno o más días, o de una o más semanas, etc., hasta el listado de su último mercado del mes. También se puede implementar con dibujos y colores, y eso permitiría trabajar con personas que no saben leer y escribir, o con niños. La forma de aplicarlo para obtener el índice está sujeta específicamente a quien haga el trabajo de facilitador de la metodología en la o las comunidades en donde se use.
A partir de este momento se inicia la etapa tres, que corresponde a la aplicación de una fórmula matemática para establecer el índice del GDA ( $i$-GDA), para lo cual se deben tener en cuenta una serie de variables (tabla 4). Considerando estas variables y los puntos totales obtenidos del producto consumido, se procede al respectivo cálculo matemático (fórmula 1) para la obtención del respectivo valor y, por ende, el $i$-GDA.

Tabla 4. Variables de la fórmula matemática del GDA

\begin{tabular}{c|l|l|}
\hline VARIABLE & \multicolumn{1}{|c|}{ DEFINICIÓN } & \multicolumn{1}{c|}{ CONSIDERACIONES DE LA VARIABLE } \\
\hline i-GDA & $\begin{array}{l}\text { Índice del globo de la } \\
\text { dependencia alimentaria }\end{array}$ & $\begin{array}{l}\text { Es le valor numérico de } 1 \text { a } 5 \text { sobre el cual se considera si la alimentación de } \\
\text { un apersona comunidad o población es local o global. }\end{array}$ \\
\hline$N$ & $\begin{array}{l}\text { Cantidad de elementos } \\
\text { (productos involucrados) }\end{array}$ & $\begin{array}{l}\text { Es la cantidad de productos involucrados en la alimentación de una persona } \\
\text { que se han considerado en el cálculo del } i \text {-GDA. }\end{array}$ \\
\hline$X$ & $\begin{array}{l}\text { Puntos resultantes de las } \\
\text { etapas } 1 \text { y } 2 \text { del modelo } \\
\text { GDA }\end{array}$ & $\begin{array}{l}\text { Es el resultado de los puntos que se suman por la colocación de cada producto, } \\
\text { ola suma de todos los puntos de todos los productos (en el caso de que sean más } \\
\text { de } 1 \text { ) ubicados en el globo, según su nivel de origen y origen de cuadrante. }\end{array}$ \\
\hline
\end{tabular}

Fuente: elaboración propia.

Fórmula 1. Cálculo del $i$-GDA a partir de los puntos obtenido en las etapas 1 y 2 de cada producto.

$$
i-G D A=\frac{N \cdot 10}{x}
$$

Fuente: elaboración propia.

En el ejemplo práctico, en el que se tomó un solo producto (el plátano, pero se pueden tomar muchos al mismo tiempo, porque se supone que la alimentación de una persona o una comunidad no se compone de un solo producto, en el mejor de los casos), el total de los puntos sumados del producto en las etapas 1 y 2 fue de 9 , así entonces queda:

$N=1$

$X=9$

Entonces, la fórmula queda:

Fórmula 2. Resultados de aplicación de la fórmula al ejemplo del plátano

$$
i-\mathrm{GDA}=\frac{1 \cdot 10}{9} \rightarrow i-\mathrm{GDA}=1,11
$$

Fuente: elaboración propia.

El índice obtenido de 1,11 se establece según la escala del modelo GDA, en consumo de base local (figura 3), que finalmente se ajusta a lo expuesto en el mismo ejemplo. Es también un indicador de que, en el caso de plátano, que es un recurso local, es parte de la alimentación de una persona de la misma localidad. Si los productos involucrados son varios, la fórmula también tendrá un rango que va de 1 a 5 , y su valor en la mayoría de los casos nunca será 1 o 5, porque siempre existen variables que no dejan que sea totalmente soberanos o locales, como el tipo de mercados, en los cuales siempre existe o un intermediario (que obliga a comprar los productos), o productos externos necesarios para la alimentación como la sal o las grasas (aceites), entre otros.

Figura 3. Escala de valoración del modelo GDA

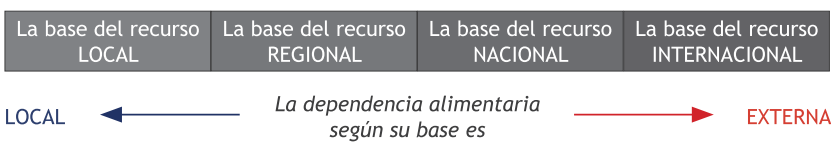

Fuente: elaboración propia.

Finalmente, el proceso por el cual se debe manejar el modelo GDA debe obligatoriamente seguir los siguientes pasos.

i. Determine el nivel de origen y el cuadrante de recorrido de cada uno de los productos -en el caso de que sean más de uno- y ubíquelo en el globo. 
ii. De acuerdo a la ubicación en el globo, asigne los puntajes de la tabla 2.

iii. De acuerdo al tipo de mercado o la forma en que adquirió o consiguió el o los productos, reste los puntos según la tabla 3 . Recuerde que la resta se hace por producto y no de forma total ${ }^{7}$ de todos los productos.

iv. Sume el total de los puntos de todos los productos y reemplace los datos en la fórmula.

v. Valore según la escala del modelo y determine todos los análisis sobre la alimentación de la persona, comunidad o región de donde se han tomado los datos para estable el $i$-GDA.

Este modelo, al igual que otros, es solo un índice que permite ver de forma global, generalizada, pero muy ubicada el origen de nuestra alimentación, lo que permite, a partir de sus resultados, conocer si nuestra comida, la que se usa en nuestra dieta diaria, es de origen local, regional, nacional o global. Esto hace posible establecer prioridades en el establecimiento de políticas, estrategias o modelos para incentivar la producción y el consumo local (PNDU, 2011).

La herramienta $i$-GDA ya se ha probado en diferentes proyectos y diferentes espacios de trabajo con comunidades, y como resultado se mencionarán algunos ejemplos de aplicaciones ya hechas, con imágenes de los talleres en dichos espacios y los resultados generales obtenidos.

\section{RESULTADOS EN PROYECTOS Y DISCUSIONES}

La metodología GDA ya se ha puesto en prueba en diferentes proyectos. En este apartado se mostrará el resultado en tres proyectos. El primero en el que se usó fue en la tesis de Cindy Pamela Quintero,

\footnotetext{
7 Siguiendo el ejemplo, y para hacer claridad sobre el proceso, vamos agregar dos productos más, con dos variables particulares. Al plátano que ya traemos como ejemplo, que es el producto 1 , se le agregan huevos, que sería el producto 2 , y arroz que sería el producto 3 . Tenemos en cuenta que los huevos son de las gallinas de su patio (los produce) y el arroz lo compró en una tienda y es originario de otro departamento, pero lejano (nacional). A cada uno de ellos se le debe aplicar la fórmula. Así, el plátano tendría 9 puntos (como ya está en el ejemplo), los huevos tendrían 10 puntos (por ser del nivel 1 en el cuadrante A, y no se le restan puntos por estar producidos en su patio), y el arroz tendría 5 puntos (por ser nacional ,4 puntos, y de un lugar lejano, 2 puntos, se le resta 1 punto por ser comprado). Entonces, el total sería $10+9+5=24$. En la fórmula quedaría: $N=$ 3 productos, $X=24$ puntos, y el resultado sería: 3 multiplicado por 10 , dividido entre 24 , es decir el $i$-GDA es de 1,25 , de base local, según la escala del modelo.
}

graduada del programa de Ingeniería Agroecológica, que trabajó su investigación en memoria biocultural con la comunidad indígena sáliba, en el departamento del Casanare, en Colombia (Quintero Corredor \& Monje Carvajal, 2012). El segundo caso es uno de los talleres sobre soberanía alimentaria en el diplomado de Agroecología y Agrobiodiversidad para el trópico húmedo que impartió UNIMINUTO a instructores del Sena ${ }^{8}$ en siete centros regionales (Quibdó, Buenaventura, Guapi, Tumaco, Leticia y Yopal), en cooperación con el Sena y Tropenbos. El tercer proyecto es un taller teórico-práctico mediado en comunidades de productores de hortalizas urbanas, específicamente con la Red de Agricultura Urbana de Usaquén, en la ciudad de Bogotá.

En la tesis de Cindy Quintero (Quintero Corredor \& Monje Carvajal, 2012), se realizó la aplicación del modelo y el resultado mostró que la etnia dependía en su mayoría del recurso local para su alimentación. Aunque los recursos que se usan son locales, estos no son propios. La comunidad, dentro de la dieta que consume, únicamente produce 8 alimentos y 16 son comprados en el municipio cercano. Los alimentos producidos o conseguidos dentro del resguardo son yuca, plátano, pescado, chigüiro (Hydrochoerus hydrochaeris), cachicamo ${ }^{9}$, mañoco (harina de yuca brava), casabe (especies de tortillas que se hacen con mañoco), limón. $Y$ entre los que son comprados se encontraron arroz, papa, granos, pasta, panela, azúcar, café, aceite, verduras, sal y chocolate. El resultado del modelo GDA en la comunidad sáliba del Suspiro en el Casanare fue de 1,6, que muestra que a pesar de que su alimentación involucra productos externos (en su mayoría nacionales), su índice es fundamentalmente de recurso local (Quintero Corredor \& Monje Carvajal, 2012).

En el diplomado de Agroecología y Agrobiodiversidad para el trópico húmedo Sena-Tropenbos, solo contaremos el taller realizado por el Ingeniero Daniel Andrés Vega, en la ciudad de Quibdó, en el departamento del Chocó, en una de las zonas rurales de la capital del departamento. Vega aplicó el modelo con los instructores del Sena que estaban tomando

8 El Servicio Nacional de Aprendizaje SENA, es una institución pública colombiana encargada de dar programas de formación terciaria, complementaria y titulada para el trabajo.

9 Mamífero endentado de América Central y Suramérica Dasypodidae, que tiene su dorso y cola protegidos por placas córneas articuladas de manera que le permiten arrollarse; es un cavador innato y se suele alimentar de invertebrados como lombrices y vegetales; su carne es muy apreciada; existen varias familias. En el caso de la sabana llanera en Colombia la especie más conocida es Dasypus sabanicola. 
el diplomado (figura 4) y usó el periodo de una semana para evaluar la herramienta.

Figura 4. Estudiantes del diplomado en taller de campo, aplicando modelo GDA

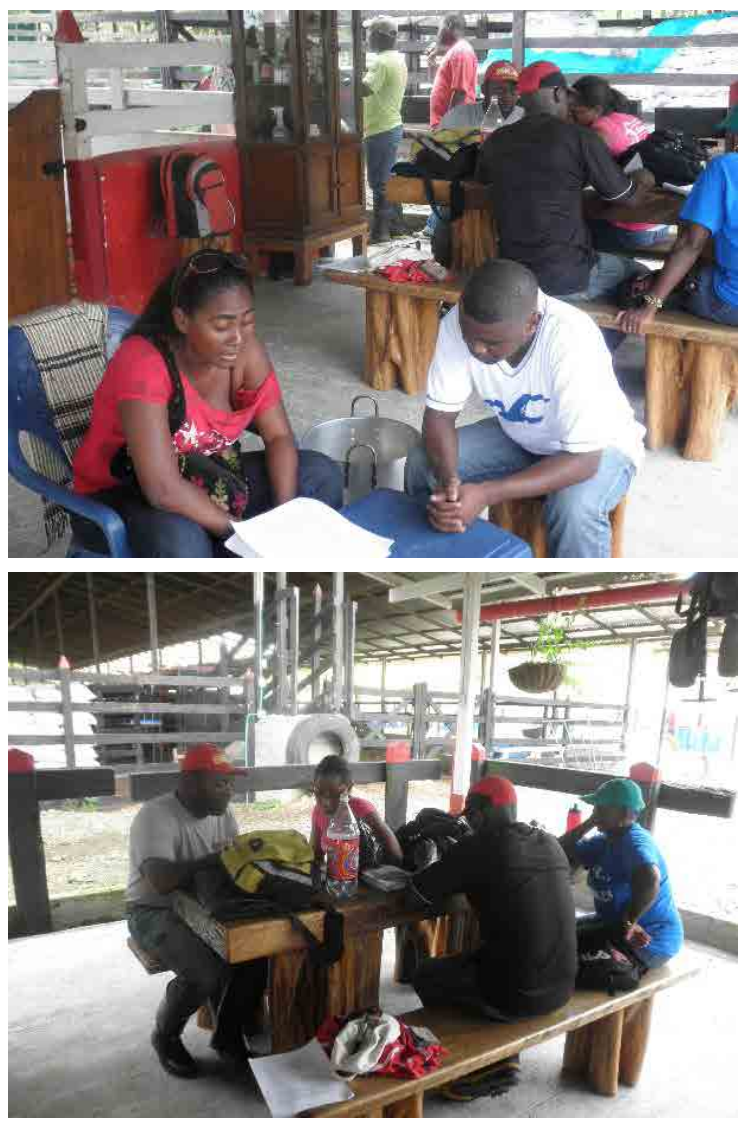

Fuente: elaboración propia.

Fotografía tomada en una jornada de campo en Quibdó, en el departamento de Chocó, en el marco del diplomado en Agroecología y Agrobiodiversidad para el Trópico Húmedo de uniminuto, en el convenio, Sena-Tropenbos, en 2011.

El resultado promedio de los estudiantes que desarrollaron por grupos la aplicación del modelo GDA fue de un $i$-GDA de 2,6; es decir, la base de alimentación es de recurso regional. La explicación de este índice fue que muchos de los productos no se producen en el departamento, y que el transporte hasta Quibdó implica distancias considerables pues sus vías de comunicación demandan tiempo y recursos para el transporte de alimentos desde las zonas en donde se producen hasta la capital del departamento del Chocó.

También fue influyente en que no fuera más alto el índice que el sistema de alimentación de la población del Chocó, la cual tiene elementos muy básicos, exóticos, que no involucra una variedad muy amplia en sus comidas. Fundamentalmente, su base alimentaria es el plátano y el pescado: el primero de estos dos se produce en el mismo departamento, y el segundo es un recurso fundamentalmente local.

En el tercer lugar en donde se ha usado experimentalmente el modelo GDA fue en un taller teórico-práctico impartido por el ingeniero Daniel Andrés Vega, el cual fue dirigido a los participantes de la Red de Agricultura Urbana de Usaquén, la cual orienta sus esfuerzos al desarrollo de planes de seguridad alimentaria y nutricional y la promoción del uso de tecnologías limpias y prácticas de desarrollo sostenible. Estos agricultores urbanos se encuentran localizados en el barrio Soratama, de la ciudad de Bogotá.

El trabajo se desarrolló con una población heterogénea, distribuida en niños, jóvenes, adultos y adultos mayores, que usaron el modelo para evaluar la alimentación de una semana. El resultado del índice fue un $i$-GDA de 3,4, el cual, según los análisis hechos en el taller, se debe a tres aspectos específicos. El primero es que la base alimentaria es la papa y el pollo, que se producen cerca de Bogotá, pero implican un transporte; el segundo es que la alimentación en Bogotá involucra muchos elementos que llegan de otros departamentos con unas distancias considerables que requieren un consumo de combustible por transporte; y tercero es que en la capital de la república nadie produce su propia comida, y el modelo de mercado es fundamentalmente de compra con dinero convencional en mercados o plazas en donde los que venden son intermediarios, lo que aumenta la cadena comercial, factor que es un indicador de baja sustentabilidad según lo propuesto por la agroecología en sus tratados. Así mismo, y aunque el índice obtenido no fue muy bajo, se resalta la importancia de la producción de hortalizas urbanas, que fomentan en gran medida grados de seguridad y soberanía alimentaria dentro de la comunidad de productores urbanos. 
Figura 5. Habitantes de la localidad de Usaquén desarrollando el modelo GDA con la Red de Agricultura Urbana
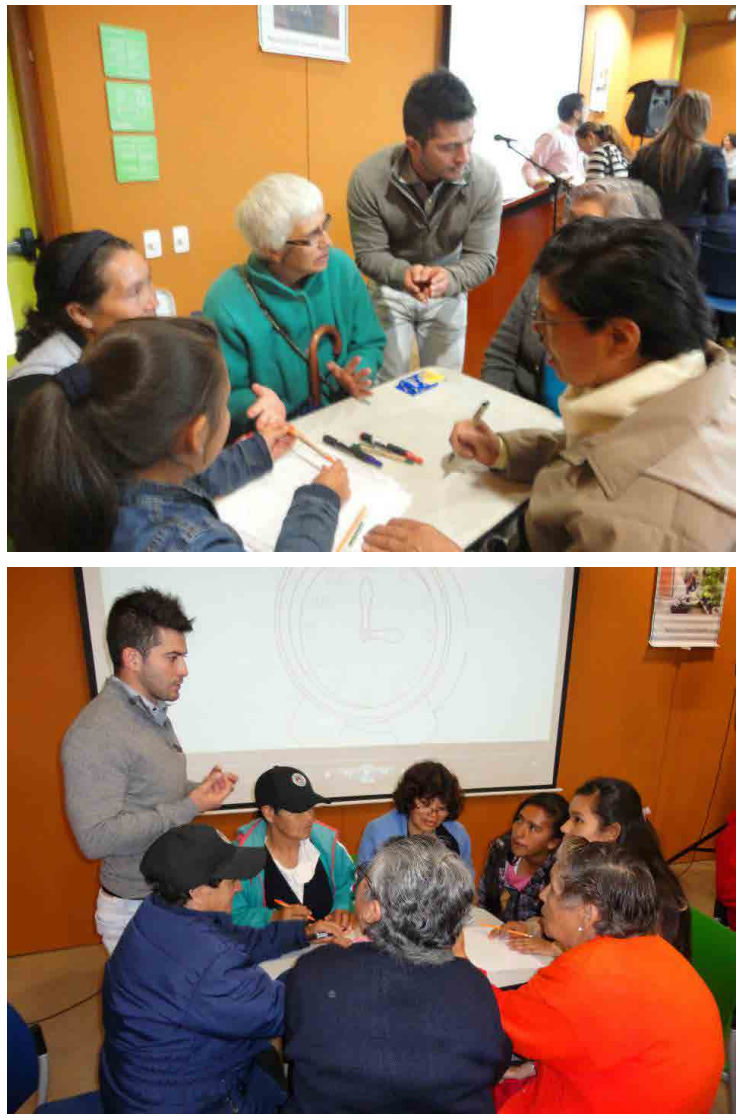

Fuente: elaboración propia.

Fotografía tomada en un taller sobre soberanía y seguridad alimentaria, en la localidad de Usaquén en el 2012, con adultos y adultos mayores de la comunidad, como estrategias para fomentar la agricultura urbana en los barrios que componen la localidad.

\section{CONCLUSIONES}

La herramienta GDA no solo permite conocer indicadores cuantitativos asociados a la seguridad y soberanía alimentaria de una persona o una comunidad, sino que también se convierte en una apuesta integradora para obtener diagnósticos participativos incluyentes, que promueven la puesta en marcha de métodos de acción para mitigar la dependencia alimentaria mediante la implementación de planes que incentiven y potencien los modelos tradicionales de producción, apropiación y consecución de alimentos locales.

Al evaluar el modelo, se logra determinar que el sitio de aplicación de esta herramienta incide considerablemente en los resultados obtenidos, dado que el modelo está ligado a las condiciones geográficas para la producción, el tipo de alimentación o costumbres que han definido las bases alimentarias en familias y comunidades del país, y que están en muchas ocasiones relacionadas con las disposiciones económicas que determinan la consecución de alimentos.

Dentro de los Objetivos del Desarrollo Sostenible sobresalen la erradicación del hambre y la vida de ecosistemas terrestres y submarinos. Esta herramienta, aplicada de forma integral y a través de un proceso de reflexión consiente, permite apalancar en gran medida el cumplimiento de las metas del milenio, por medio de estrategias que propicien de manera sustentable todos los eslabones involucrados en la cadena productiva de alimentos agropecuarios, haciendo hincapié en el aprovechamiento del recurso local como eje fundamental de la producción y el consumo responsable, la circulación alternativa de bienes y servicios, que repercutan de manera positiva sobre la seguridad, la soberanía y la suficiencia alimentaria.

Asimismo, a la fecha se pueden encontrar instituciones que trabajan con bases de datos estadísticas, que logran hacer aproximaciones sobre el consumo de alimentos por regiones específicas, pero que por su naturaleza generalizada no permiten obtener resultados puntuales que hagan posible adoptar medidas asociadas con la seguridad y soberanía alimentaria de una comunidad, familia o persona.

Por otro lado, es de resaltar que se requiere la construcción de bases de datos de alimentos con zonas de producción y tiempos de transporte, que permitan ser integradas al modelo GDA y así fortalecer la herramienta y disminuir los posibles sesgos en los resultados obtenidos.

\section{REFERENCIAS}

Alonso Mielgo, A. M., \& Sevilla Guzmán, E. (1995). El discurso ecotecnocrático de la sostenibilidad. Agricultura y desarrollo sostenible, 1-24.

Dabat, A. (2002). Globalización, capitalismo actual y nueva configuración espacial del mundo. En Basave, Dabat, Morera, Rivera, \& Rodríguez., Globalización y alternativas incluyentes para el siglo XXI (pp. 1-44). México: CRIM-UNAM.

Figueroa Pedraza, D. (2003). Seguridad alimentaria familiar. Revista saludo pública y nutrición, 4(2), 1-9. 
Gisbert Quero, J. (2013). Vivir sin empleo: Trueque, bancos de tiempo, monedas sociales y otras alternativas. Barcelona: Editorial Los Libros del Lince.

Godard, O., Ceron, J. P., Vinaver, K., \& Passaris, S. (1987). Desarrollo endógeno y diferenciación de espacios de desarrollo: Un esquema de análisis para el desarrollo local. Estudios territoriales, 135-147.

Kalmanovitz, S. (2003). Economía y nación: una breve historia de Colombia. Bogotá: Editorial Norma.

Leff, E. (2002). Saber ambiental, sustentabilidad, racionalidad, complejidad y poder. Ciudad de México: Editorial Siglo xxı.

Martí Sanz, N. (2006). La Multidimensionalidad de los sistemas locales de alimentación en los Andes peruanos [Tesis Doctoral]. Universitat Autònoma de Barcelona, Facultat de Ciències Econòmiques i Empresarials, Departament d'Economia i d'Història Econòmica, Barcelona.
Morin, E. (1999). Siete saberes necesarios para la educación del futuro. París: unESCO.

Odum, E. P., \& Barrett, G. W. (2008). Fundamentos de ecología. México: Thomson Learning Iberoamérica.

PNUD. (2011). Colombia rural: razones para la esperanza. Informe Nacional de Desarrollo Humano. Bogotá: Programa de las Naciones Unidas para el Desarrollo.

Quintero Corredor, C. P., \& Monje Carvajal, J. J. (2012). La recuperación de conocimiento tradicional de la etnia sáliba del resguardo el suspiro, municipio de Orocué Casanare, sobre su memoria biocultural, referente a la comida, la cultura y la espiritualidad [Tesis de pregrado]. Corporación Universitaria Minuto de Dios - UNIMINUTO, Bogotá.

Tocancipá, J. (2008). El trueque: tradición, resistencia y fortalecimiento de la economía indígena en el Cauca. Revista de estudios sociales(31), 146-161. 